\title{
PORT-CONTACT SYSTEMS IN BILATERAL TELEMANIPULATION
}

\author{
C. Secchi ${ }^{*}$ S. Stramigioli ${ }^{* *}$ C. Fantuzzi ${ }^{*}$ \\ * DISMI, University of Modena and Reggio Emilia, \\ Viale Allegri 13, 42100 Reggio Emilia, Italy \\ e-mail: \{secchi.cristian, fantuzzi.cesare\}@unimore.it \\ ** EEMCS, University of Twente \\ P.O.Box 217, 7500 AE Enschede, NL \\ S.Stramigioli@ieee.org
}

\begin{abstract}
In this paper we develop one of the first control applications of the recently proposed port-contact framework. We show how it is possible to model and control a bilateral telemanipulation system using port-contact systems and we develop a port-contact impedance controller that allows to impose a desired interactive behavior and a zero steady state position error during contact tasks.
\end{abstract}

Keywords: Telemanipulation, passivity, nonlinear control.

\section{INTRODUCTION}

The port-Hamiltonian framework (van der Schaft 2000) has been introduced as a generalization of Hamiltonian systems and it allows to build energy based models and controllers for physical systems. A physical system is modeled as a set of energy processing elements (i.e. energy storing, energy dissipating and energy injecting elements) that exchange energy along a power preserving interconnection. The amount of energy stored in a certain configuration is represented by a real valued Hamiltonian function $H(\cdot)$ on the state manifold $\mathcal{N}$ of energy variables while the power preserving interconnection is represented by a geometric object called Dirac structure which is defined as a linear sub-bundle. Dirac structure can depend only on the state variable and they allow to model only reversible energy transfers. Dissipative effects, namely irreversible energy transfers, are modeled by terminating some power ports of the Dirac structure with energy absorbing elements that are characterized by no state. This problem limits the modeling power of port-Hamiltonian systems. In fact, it is not possible to model irreversible energy storage phenomena as, for example, the entropy storage that takes place in Thermodynamical processes. Recently, the so-called port-contact framework (Eberard et al. 2005a, Eberard et al. 2005b) has been introduced as a generalization of the port-Hamiltonian framework for modeling thermodynamical systems. It strictly contains portHamiltonian systems and it allows to model irreversible energy tranfers between the elements composing a physical system. The structure of port-Hamiltonian systems has been extensively exploited for building energy based controllers whose structure is again port-Hamiltonian. In particular, in robotics, port-Hamiltonian systems have been used for controlling the interaction between a robot and the environment. From this point of view, port-Hamiltonian controllers can be thought as virtual mechanical systems which impose a desired impedance in the interaction (Stramigioli et al. 2005). In (Stramigioli et al. 2002, Stramigioli et al. 2005) a port-Hamiltonian bilateral telemanipulation scheme has been proposed. Both master and slave robots are modeled 
as port-Hamiltonian systems which are controlled by port-Hamiltonian impedance controllers. Master and slave sides are interconnected by a scattering based communication channel (Anderson and Spong 1989, Niemeyer and Slotine 1991) which guarantees a lossless interconnection independently of the communication delay. When using a scattering based communication channel in port-Hamiltonian telemanipulation, it happens that during contact tasks there is a mismatch between the steady state positions of master and slave. In (Secchi et al. 2006) some irreversibe energy transfers have been introduced in the controller for compensating this position error.

In this paper we provide one of the first control applications of port-contact systems. We consider a port-Hamiltonian based telemanipulation system and we show how it can be modeled using port-contact systems. Furthermore, we build a port-contact controller that allows to implement a desired impedance on the slave side and to compensate the position error arising at steady state during contact tasks, generalizing what has been proposed in (Secchi et al. 2006). The paper is organized as follows: in Sec. 2 some background on port-contact systems and bilateral telemanipulation are given; in Sec. 3 we show how it is possible to represent a port-Hamiltonian based bilateral telemanipulator in terms of port-contact systems and in Sec. 4 we modify the port-contact controller in order to be able to compensate the steady state position error. In Sec. 5 we draw some conclusions and we address some future work.

\section{BACKGROUND}

Port-contact systems. We shall present a brief overview of port-contact systems for modeling physical s ystems; for a more detailed description see (Eberard et al. 2005 a, Eberard et al. 2005b). Let $\mathcal{N} \ni x$ be the $n$-dimensional state manifold of energy variables. We can define the associated thermodynamical phase space $\mathcal{M}=\mathbb{R} \times$ $T^{*} \mathcal{N} \ni\left(x^{0}, x, p\right)$, where $T^{*} \mathcal{N}$ indicates the cotangent bundle of $\mathcal{N}$. The thermodynamical phase space contains both energy variables (e.g. momentum, charge, displacement) which live on $\mathcal{N}$, and co-energy variables (e.g. velocity, voltage, elastic force), which live on $T_{x}^{*} \mathcal{N}$ and the internal energy $x^{0}$. In $\mathcal{M}$ there is all what is necessary for describing the dynamics of any physical system. In the following we will indicate with $x^{i}$ and $p_{i}$, where $i=1, \ldots, n$, the coordinates of $x$ and $p$ respectively. It can be shown that $\mathcal{M}$ is a $2 n+1$ dimensional manifold endowed with a canonical contact form $\vartheta$ which, using canonical coordinates, can be expressed as $\vartheta=d x^{0}-\sum_{i=1}^{n} p_{i} d x^{i}$. The role of $\vartheta$ is that of setting up a relation among the internal energy, the energy and the co-energy variables which defines the physical properties of a system. It is often more convenient to express physical properties in an integral form. A Legendre submanifold of the $2 n+1$-dimensional contact manifold $\mathcal{M}$ is a $n$-dimensional submanifold of $\mathcal{M}$ which is an integral manifold of $\vartheta$. Loosely speaking a Legendre submanifold is the region of the thermodynamical phase space where a physical system satisfying certain physical properties is constrained to evolve. For a given set of coordinates, a Legendre submanifold is determined by a generating function $F: T^{*} \mathcal{N} \mapsto \mathbb{R}$ with $n$ arguments. In the particular case in which $F: \mathcal{N} \mapsto \mathbb{R}$, the Legendre submanifold is the set

$$
\mathcal{L}_{F}=\left\{F, x^{1}, \ldots, x^{n}, p_{1}=\frac{\partial F}{\partial x^{1}}, \ldots, p_{n}=\frac{\partial F}{\partial x_{n}}\right\}
$$

A contact vector field over $\mathcal{M}$ is a vector field which preserves the contact structure $\vartheta$. There exists an isomorphism between contact vector fields and differentiable functions over $\mathcal{M}$. Given a generating function $f$, called contact Hamiltonian, the corresponding contact vector field will be indicated by $X_{f}$. Using coordinates $X_{f}$ can be expressed as:

$$
\begin{array}{r}
X_{f}=\left(f-\sum_{k=1}^{n} p_{k} \frac{\partial f}{\partial p_{k}}\right) \frac{\partial}{\partial x^{0}}+\frac{\partial f}{\partial x^{0}}\left(\sum_{k=1}^{n} p_{k} \frac{\partial}{\partial p_{k}}\right)+ \\
+\sum_{k=1}^{n}\left(\frac{\partial f}{\partial x^{k}} \frac{\partial}{\partial p_{k}}-\frac{\partial f}{\partial p_{k}} \frac{\partial}{\partial x^{k}}\right)
\end{array}
$$

Furthermore, given two function $f, g: \mathcal{M} \mapsto \mathbb{R}$ it is possible to define the following Jacobi brackets which, in coordinates are defined as:

$$
\begin{array}{r}
\{f, g\}=\sum_{k=1}^{n}\left(\frac{\partial f}{\partial x^{k}} \frac{\partial g}{\partial p_{k}}-\frac{\partial g}{\partial x^{k}} \frac{\partial f}{\partial p_{k}}\right)+(f- \\
\left.-\sum_{k=1}^{n} p_{k} \frac{\partial f}{\partial p_{k}}\right) \frac{\partial g}{\partial x^{0}}-\left(g-\sum_{k=1}^{n} p_{k} \frac{\partial g}{\partial p_{k}}\right) \frac{\partial f}{\partial x^{0}}
\end{array}
$$

When using contact vector fields for describing the dynamics of a physical system, it is necessary that they leave the Legendre submanifold, capturing the physical properties of the system, invariant. It can be proven that a contact vector field $X_{f}$ leaves invariant a Legendre submanifold $\mathcal{L}$ if and only if $f$ is identically zero on $\mathcal{L}$, (Eberard et al. 2005b).

A control contact system is defined by a strictly contact manifold $(\mathcal{M}, \theta)$, a Legendre submanifold $\mathcal{L}$ of $\mathcal{M}$, an input space $\mathcal{U}=\mathbb{R}^{m}$ and input functions $u_{i}, i=1, \ldots m ; m+1$ contact Hamiltonians: $K_{0}$ the internal contact Hamiltonian and $K_{j}$ the interaction Hamiltonians, satisfying the invariance condition $K_{j \mid \mathcal{L}}=0$ for $j=0, \ldots, m$. The dynamics of the system is given by the following differential equation

$$
\frac{d}{d t}\left(x^{0}, x, p\right)=X_{K_{0}}+\sum_{j=1}^{m} u_{j} X_{K_{j}}
$$


Given a function $V$ on $\mathcal{M}$, it is possible to define the $V$-conjugated output variable $y_{V}$ as

$$
y_{V}^{j}=\left\{K_{j}, V\right\}+V \frac{\partial K_{j}}{\partial x^{0}} \quad j=1, \ldots, m
$$

and a source term $s_{V}$ as

$$
s_{V}=\left\{K_{0}, V\right\}+V \frac{\partial K_{0}}{\partial x^{0}}
$$

If $s_{V \mid \mathcal{L}}=0$, then $V$ is a conserved quantity of a conservative control contact system, in the sense that

$$
\frac{d V}{d t}=\sum_{j=1}^{m} u_{j} y_{V}^{j}
$$

A port-contact system is a conservative control contact system such that the generating function of the Legendre submanifold is a conserved quantity. In this case, we have that the dynamics of the system restricted to the Legendre submanifold is lossless.

Port-Hamiltonian based bilateral telemanipulation. Port-Hamiltonian systems are defined on the space of energy variables $\mathcal{N}$. They are characterized by an Hamiltonian energy function, expressing the stored energy, and by a Dirac structure $\mathcal{D}$, representing the internal energetic interconnections. Using coordinates, in their simplest form, they are represented by the following equations

$$
\left\{\begin{array}{l}
\dot{x}=(J(x)-R(x)) \frac{\partial H_{0}}{\partial x}+\sum_{j=1}^{m} u_{j}(t) g_{j}(t) \\
y^{j}=g_{j}^{T} \frac{\partial H_{0}}{\partial x} \quad j=1, \ldots, m
\end{array}\right.
$$

where $J(x)$ is a skew-symmetric matrix representing the Dirac structure, $R(x)$ is a positive semidefinite function representing the energy dissipated by the system, $H_{0}$ is the Hamiltonian function, $u=\left(u_{1}, \ldots, u_{m}\right)^{T}$ is the input vector. $g_{1}, \ldots, g_{m}$ are the input vector fields on $\mathcal{N}$ and $y=\left(y^{1}, \ldots, y^{m}\right)^{T}$ is the conjugated output variable. It can be easily seen (van der Schaft 2000) that a port-Hamiltonian system is passive with respect to $H_{0}$. Thanks to their passivity and to their capability of describing both linear and nonlinear electro-mechanical systems, port-Hamiltonian systems have been quite extensively used for modeling and controlling interactive robotic devices as for example telemanipulation systems. A portHamiltonian based bilateral telemanipulation system is reported in Fig. 1 in a bond-graph notation. The system is made up of a local robot (called master) and of a remote robot called slave. Both robots can be modeled as port-Hamiltonian systems. The slave robot is interconnected to a port-Hamiltonian impedance controller whose role is to regulate the interaction of the slave with the remote environment. A human operator can interact with the master whose motion is transmitted to the remote side in order to move the

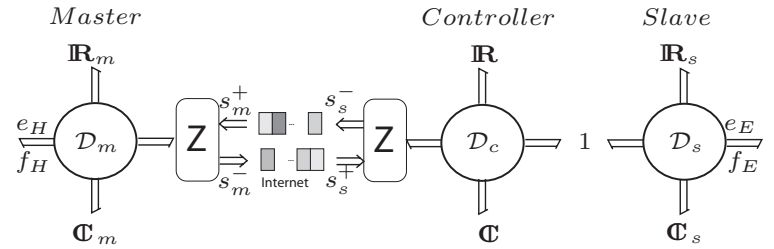

Fig. 1. The Port-Hamiltonian based Telemanipulation Scheme.

slave accordingly. When the slave interacts with the remote environment, the interaction force is fed back to the master side in order to improve the feeling of the remote interaction perceived by the user. Master and slave side exchange force and velocity information through a scattering based communication channel (Anderson and Spong 1989, Niemeyer and Slotine 1991, Stramigioli et al. 2002) which is characterized by a lossless behavior independently of any constant communication delay. The overall telemanipulation system consists of the interconnection of passive master and slave sides through a lossless communication channel and, therefore, it is a passive system characterized by a stable behavior both in free motion and when interacting with the remote environment.

The controller can be interpreted as a virtual physical system imposing the desired impedance on the slave; it always contains at least one elastic element used to set the stiffness of the interaction. The scattering based communication channel can be interpreted as a distributed mass-spring system and it implements a virtual mechanical coupling between master and slave sides. As reported in (Niemeyer 2004), during contact tasks, the force feedback is transmitted to the slave side through a virtual deformation of the distributed system representing the communication channel. This gives rise to a difference in the steady state positions of master and slave which is equal to $\Delta=T Z^{-1} e_{E}$, where $T$ is the communication delay, $Z$ is the positive definite impedance of the scattering transformation and $e_{E}$ is the contact force between the slave and the environment. In (Secchi et al. 2006), in order to solve this problem, a modification to the port-Hamiltonian controller at the slave side has been proposed. Shortly, the dissipative element used to match the impedance of the scattering transformation, has been replaced with an energy storing element called tank and a modification to the Dirac structure of the controller has been made in order to force an energy absorbing behavior of the tank. Furthermore, the interconnection structure of the controller has been modified to create a controllable energy transfer between the tank and the rest length characterizing one of the elastic elements of the controller. By driving energy form the tank to the rest length it is possible to introduce an 
offset that compensates the position error taking place between master and slave.

\section{PORT-CONTACT BASED BILATERAL TELEMANIPULATION}

In this section we will show how it is possible to model a port-Hamiltonian based bilateral telemanipulation using port-contact systems. Furthermore, generalizing the approach proposed in (Eberard et al. 2005b, Secchi et al. 2006) and exploiting the properties of port-contact systems, we will model the energy dissipation that is present in port-Hamiltonian systems as an irreversible storage of energy that will be used, as it will be shown in the next section, for passively compensating the steady state position error between master and slave robots during contact tasks.

A port-Hamiltonian system with dissipation can be modeled as a port-contact system characterized by an irreversible energy storage phenomenon. Let us considered a port-Hamiltonian system defined on the manifold of energy variables $\mathcal{N}$ and described by Eq.(8). In order to build the portcontact representation of the system we follow the approach proposed in (Eberard et al. 2005b) and we firstly define the extended base space $\mathcal{N}_{e}$ of energy variables as $N_{e}=\mathcal{N} \times \mathbb{R}$. Using the same notation of (Secchi et al. 2006), the extra energy variable added will model the state of a tank where the energy that would be dissipated using the port-Hamiltonian framework is irreversibly stored. In other words, it plays the same role as entropy in physical systems where dissipative phenomena correspond to an increase of entropy. It is now possible to define the thermodynamical phase space on which to build the port-contact system as

$$
\mathcal{M}=\mathbb{R} \times T^{*} \mathcal{N}_{e} \ni\left(x^{0}, x^{1}, \ldots, x^{n}, x^{t}, p_{1}, \ldots, p_{n}, p_{t}\right)
$$

where the index $t$ denotes the energy and the co-energy variable associated to the tank. The contact form associated to $\mathcal{M}$ is given by $\vartheta=$ $d x^{0}-\sum_{i=1}^{n} p_{i} d x^{i}-p_{t} d x^{t}$. We can describe the Legendre submanifold that defines the relation between energy variables, co-energy variables and internal energy, using as a generating function the internal energy function $H_{e}: \mathcal{N}_{e} \mapsto \mathbb{R}$ defined as

$$
H_{e}\left(x, x^{t}\right)=H_{0}(x)+H_{t}\left(x^{t}\right)
$$

where the first term describes the reversible energy storage phenomena modeled in Eq.(8) and the second term models the irreversible energy storage corresponding to the dissipative phenomena modeled in Eq.(8). The Legendre manifold is defined by

$$
\begin{aligned}
\mathcal{L}= & \left\{H_{e}\left(x, x^{t}\right), x^{1}, \ldots, x^{n}, x^{t},\right. \\
& \left.p_{1}=\frac{\partial H_{e}}{\partial x_{1}}, \ldots, p_{n}=\frac{\partial H_{e}}{\partial x^{n}}, p_{t}=\frac{\partial H_{e}}{\partial x^{t}}\right\}
\end{aligned}
$$

We can now define the $m+1$ contact Hamiltonians as

$$
\begin{aligned}
K_{0} & =-p^{T}[J(x)-R(x)] \frac{\partial H_{e}}{\partial x}-\frac{p_{t}}{\frac{\partial H_{e}}{\partial x^{t}}} \frac{\partial^{T} H_{e}}{\partial x} R(x) \frac{\partial H_{e}}{\partial x} \\
K_{j} & =\left(\frac{\partial H_{e}}{\partial x}-p\right)^{T} g_{j}(x) \quad j=1, \ldots, m
\end{aligned}
$$

where $p=\left(p_{1}, \ldots, p_{n}\right)^{T}$ and $x=\left(x^{1}, \ldots, x^{n}\right)^{T}$. It can be easily seen that $K_{0}$ and $K_{1}, \ldots, K_{m}$ vanish on $\mathcal{L}$ and that therefore the corresponding vector fields leave invariant the Legendre submanifold and consequently the physical properties characterizing the system. Using Eq.(4) and Eq.(2) we can easily compute the dynamics on the extended space of energy variables restricted to $\mathcal{L}$ :

$$
\left\{\begin{array}{l}
\dot{x}=[J(x)-R(x)] \frac{\partial H_{0}}{\partial x}+\sum_{j=1}^{m} g_{j}(x) u_{j}(t) \\
\dot{x}^{t}=\frac{1}{\frac{\partial H_{t}}{\partial x^{t}}} \frac{\partial^{T} H_{0}}{\partial x} R(x) \frac{\partial H_{0}}{\partial x}
\end{array}\right.
$$

We can see that the dynamics of the state variables over which the port-Hamiltonian system is defined is exactly the same as that of Eq.(8) and this means that the port-contact system we are building can represent the dynamics of a portHamiltonian system with dissipation; in particular, both master and slave robots can be represented by port-contact systems and the interactive behavior imposed by the impedance controller can be exactly reproduced using a portcontact impedance controller. Furthermore, we have that the energy that was dissipated by the port-Hamiltonian system is now stored in the tank and the variation of this storage is represented by the variable $x_{t}$. In fact, formally, we have that

$$
\dot{H}_{t}=\frac{\partial H_{t}}{\partial x^{t}} \dot{x}^{t}=\frac{\partial^{T} H_{0}}{\partial x} R(x) \frac{\partial H_{0}}{\partial x}
$$

The two dynamics represented in Eq.(13) are coupled using co-energy variables and, therefore, the resulting system cannot be represented as a port-Hamiltonian system since Dirac structures cannot represent this kind of interconnection; see (Eberard et al. 2005a) for further details. In order to define the define a port-contact system we need to introduce the output variable and to show that the total energy is conserved. Using Eq.(5) with $V=H_{e}\left(x, x^{t}\right)$ and $K_{0}$ and $K_{j}$ defined as in Eq.(12), it is possible to see that the conjugated output is

$$
y^{j}=g_{j}^{T}(x) \frac{\partial H_{0}}{\partial x} \quad j=1, \ldots, m
$$

namely, exactly the same output of Eq.(8). Using this output and Eq.(6) it can be seen that the source term is zero on all the thermodynamical phase space and that therefore the total energy $H_{e}$ is conserved and, consequently, the system is lossless.

Thus, we have that all the port-Hamiltonian systems present in Fig. 1 can be modeled as port- 
contact systems. Since all the systems in Fig. 1 are interconnected by means of power preserving interconnections and since the power preserving interconnection of two port-contact systems is again a port-contact system (Eberard et al. $2005 b$ ), we have that the overall telemanipulation system is lossless and, consequently, passive. We can build an intrinsically passive bilateral telemanipulation system using port-contact system. We can achieve exactly the same dynamic behavior that we have when using port-Hamiltonian system and, therefore, the storage of the energy that would be dissipated by port-Hamiltonian systems is completely invisible to the user who does not perceive any spurious behavior when using the telemanipulator. Thanks to the portcontact structure, we have that the energy that was dissipated is now stored in a tank. In the case of master and slave robots, the tank just represent the increase of some entropy-like energy variable due to dissipative phenomena. In the case of the impedance controller, we can use this reserve of energy to modify the state of the controller without affecting its passive behavior and, therefore, without destabilizing the telemanipulation system. The definition of $H_{e}$ give in Eq.(10) generalizes that given in (Eberard et al. $2005 b)$ where the co-energy variable associated to the irreversible energy storage was constant. This is because the main goal of (Eberard et al. 2005b) was to model systems arising from thermodynamics and NOT the use of port-contact systems for control purposes. Using Eq.(10) we allow a greater flexibility in the way in which the energy dissipated is stored which depends on the particular choice of $H_{t}$. The port-contact controller derived using the construction developed in this section generalizes also the results of (Secchi et al. 2006) where only a part of the energy dissipated by the port-Hamiltonian controller is stored.

\section{NONLINEAR COUPLINGS AND THE POSITION ERROR COMPENSATION}

In this section, we modify the port-contact controller used in the scheme developed in the previous section in order to use the energy stored in $x^{t}$ for changing the rest length of one of the elastic elements of the controller. Consider the port-contact system modeling the dynamics of a port-Hamiltonian impedance controller, whose dynamics is described by the equations Eq.(8), and storing in $x^{t}$ the energy dissipated by the port-Hamiltonian dynamics. Since we just want to modify the internal interconnections of the controller, both the input contact Hamiltonians $K_{1}, \ldots, K_{m}$ and the output of the systems will remain the same as those proposed in Eq.(12). What we need to change is the internal contact
Hamiltonian $K_{0}$. In order to use energy for changing its value, we need to model the rest length of one of the elastic elements of the system, which is usually described by a constant parameter, as an energy variable and, therefore, we need to further extend the base space of energy variables $\mathcal{N}_{e}$ defined in the previous section and to define $\mathcal{N}_{l}=\mathcal{N}_{e} \times \mathcal{X}_{l}$.where $\mathcal{X}_{l}$ is the $l$-dimensional manifold on which the energy variable representing the rest length lives. Proceeding as in Sec. 3, we can define the thermodynamical phase space as

$\mathcal{M}_{l}=\mathbb{R} \times T^{*} \mathcal{N}_{l} \ni\left(x^{0}, x^{1}, \ldots, x^{n}, x^{t}, x^{l}, p_{1}, \ldots, p_{n}, p_{t}, p_{l}\right)$

which is characterized by the following contact form $\vartheta=d x^{0}-\sum_{i=1}^{n} p_{i} d x^{i}-p_{t} d x^{t}-p_{l} d x^{l}$ The internal energy function is $H_{e}\left(x, x^{l}, x^{t}\right)=$ $H_{0}\left(x, x^{l}\right)+H_{t}\left(x^{t}\right)$. Now the rest length enters as an argument of the energy function and not simply as a parameter. Using $H_{e}$ as a generating function, it is possible to determine the Legendre submanifold as:

$$
\begin{gathered}
\mathcal{L}=\left\{H_{e}\left(x, x^{t}, x^{l}\right), x^{1}, \ldots, x^{n}, x^{t}, x^{l},\right. \\
\left.\left.p_{1}=\frac{\partial H_{e}}{\partial x^{1}}, \ldots, p_{n}=\frac{\partial H_{e}}{\partial x^{n}}, p_{t}=\frac{\partial H_{e}}{\partial x^{t}}, p_{l}=\frac{\partial H_{e}}{\partial x_{l}}\right)\right\}
\end{gathered}
$$

Now we want to extend the internal contact Hamiltonian $K_{0}$ in order to energetically couple $x^{t}$ and $x^{l}$ in a controllable way while preserving the losslessness of the controller; in this way part of the energy stored in the can be used to change $x^{l}$. Consider the following internal contact Hamiltonian:

$$
\begin{aligned}
& K_{0}=-p^{T}[J(x)-R(x)] \frac{\partial H_{e}}{\partial x}-\frac{p_{t}}{\frac{\partial H_{e}}{\partial x^{t}}} \frac{\partial^{T} H_{e}}{\partial x^{t}} R(x) \frac{\partial H_{e}}{\partial x}- \\
& -p_{t} A \frac{\partial H_{e}}{\partial x^{l}}+p_{l}^{T} A^{T} \frac{\partial H_{e}}{\partial x^{t}}-p_{l}^{T} J_{1} \frac{\partial H_{e}}{\partial x^{l}}
\end{aligned}
$$

where $p=\left(p_{1}, \ldots, p_{n}\right)^{T}, x=\left(x^{1}, \ldots, x^{n}\right)^{T}, A$ is a matrix of proper dimensions and $J_{1}$ is a skewsymmetric matrix. Both $A$ and $J_{1}$ can be freely chosen. It can be easily seen that $K_{0 \mid \mathcal{L}}=0$. Using Eq.(18) for defining $X_{K_{0}}$ and using the same interaction contact Hamiltonians proposed in Eq.(12) for defining the $X_{K_{j}}$ and by keeping the same output used in Eq.(15), we obtain a portcontact system whose dynamics projected on $\mathcal{N}_{l}$ is given by:

$$
\left\{\begin{array}{l}
\dot{x}=[J(x)-R(x)] \frac{\partial H_{0}}{\partial x}+\sum_{j=1}^{m} g_{j}(x) u_{j}(t) \\
\dot{x}^{t}=\frac{1}{\frac{\partial H_{e}}{\partial x^{t}}} \frac{\partial^{T} H_{0}}{\partial x} R(x) \frac{\partial H_{0}}{\partial x}+A \frac{\partial H_{0}}{\partial x^{l}} \\
\dot{x}^{l}=-A^{T} \frac{\partial H_{e}}{\partial x^{t}}-J_{1} \frac{\partial H_{e}}{\partial x^{l}}
\end{array}\right.
$$

The dynamics of the energy variables $x^{1}, \ldots, x^{n}$ is the same as that imposed by the port-Hamiltonian controller, the energy dissipated by the portHamiltonian dynamics is stored in the tank whose state is described by the energy variable $x^{t}$. We 
now can see that, by properly setting $A$ and $J_{1}$ we can change $x^{l}$ and, therefore, we can impose an offset that compensates the steady state position error arising during contact tasks. Using the internal contact-Hamiltonian reported in Eq.(18) we have built a power preserving interconnection between the energy variables $x^{t}$ and $x^{l}$. In fact, we have that

$$
\dot{H}_{e}=\sum_{k=1}^{n} \frac{\partial^{T} H_{e}}{\partial x^{i}} \dot{x}^{i}+\underbrace{\frac{\partial^{T} H_{e}}{\partial x^{t}} \dot{x}^{t}}_{\dot{H}^{t}}+\underbrace{\frac{\partial^{T} H_{e}}{\partial x^{l}} \dot{x}^{l}}_{\dot{H}_{l}}
$$

Furthermore we can write

$$
\dot{H}_{t}=\frac{\partial^{T} H_{0}}{\partial x} R(x) \frac{\partial H_{0}}{\partial x}+\frac{\partial^{T} H_{e}}{\partial x^{t}} A \frac{\partial H_{e}}{\partial x^{l}}=\dot{H}_{t 1}+\dot{H}_{t 2}
$$

and

$$
\dot{H}_{l}=\frac{\partial^{T} H_{e}}{\partial x^{l}}\left(-A^{T} \frac{\partial H_{e}}{\partial x^{t}}-J_{1} \frac{\partial H_{e}}{\partial x^{l}}\right)=-\dot{H}_{t 2}
$$

The energy supplied by the variation of $x^{l}$ is equal to that extracted from the energy tank. Thanks to this energy balance, the total energy of the system remains the same. In order to regulate the variation of $x^{l}$ independently of the behavior of the system, we need to design $A$ and $J_{1}$ in order to be able to control the energy flow between the tank and $x^{l}$. A possible choice is the following, which is a generalization of the interconnection proposed in (Secchi et al. 2006):

$$
A=\Gamma \frac{\partial H_{e}}{\partial x^{l}} \frac{\partial H_{e}}{\partial x^{t}} \quad J_{1}=0
$$

where $\Gamma$ is a $l \times l$ diagonal matrix whose diagonal entries are $\gamma_{1}, \ldots, \gamma_{l}$. In this way

$$
\dot{H}_{l}=-\frac{\partial^{T} H_{e}}{\partial x^{l}} \Gamma \frac{\partial H_{e}}{\partial x^{l}}\left(\frac{\partial H_{e}}{\partial x_{t}}\right)^{2}
$$

Setting $\gamma_{i}>0$, for $i=1, \ldots, l$ we always supply energy to the tank and setting $\gamma_{i}<0$ for $i=1, \ldots, l$ we always extract energy from the tank. The magnitude of $\gamma_{i}$ can be used to boost the energy transfer. We are interested in changing the value of $x^{l}$ and, therefore, when setting the values of the entries of $\Gamma$ we have to monitor the value of $\frac{\partial H_{e}}{\partial x^{t}}$. If we want that the $i^{t h}$ component of $x^{l}$ increases, we have to set $\gamma_{i}>0$ if the $i^{\text {th }}$ component of $\frac{\partial H_{e}}{\partial x^{l}}$ is negative and $\gamma_{i}<0$ otherwise. Similar considerations hold in case we want to decrease a component of $x^{l}$. A possible algorithm for determining the sign and the magnitude of the elements of $\Gamma$ for compensating the steady state position error can be found in (Secchi et al. 2006). Notice that the choice reported in Eq.(23) introduces a coupling between the dynamics of $x^{t}$ and of $x^{l}$ that depend both on energy and coenergy variables. This interconnection is the key for being able to control the transfer of energy between $x^{l}$ and $x^{t}$ using $\Gamma$ and it cannot be modeled using Dirac structures. Thus, using contact systems it is possible to embed irreversible energy transfers in the controller which can be used for compensating the steady state position error. The controller proposed in Eq.(19) generalizes the one proposed in (Secchi et al. 2006) and it provides a greater flexibility in the design of the coupling between $x^{l}$ adn $x^{t}$.

\section{CONCLUSIONS}

In this paper we have shown the benefits of the port-contact framework in the control of a bilateral telemanipulation system. Thanks to the port-contact structure, it has been possible to store the energy that was dissipated by the portHamiltonian controller and to use to compensate an undesired steady state position error. For a matter of space it has not been possible to insert the simulations that validate the controller. Future work will investigate other possible choices of $A$ and $J_{1}$ for shaping the port-contact controller in bilateral telemanipulation and the use of the portcontact framework for controlling physical system.

\section{REFERENCES}

Anderson, R. and M. Spong (1989). Bilateral control of teleoperators with time delay. IEEE Transactions on Automatic Control 34(5), 494-501.

Eberard, D., B. Maschke and A.J. van der Schaft $(2005 a)$. Conservative systems with ports on contact manifolds. In: Proceedings of IFAC world Congress. Praha, Czech Republic.

Eberard, D., B. Maschke and A.J. van der Schaft $(2005 b)$. Port contact systems for irreversible thermodynamical systems. In: Proceedings of IEEE CDC. Seville, Spain.

Niemeyer, G. and J. Slotine (1991). Stable adaptive teleoperation. IEEE Journal of Oceanic Engineering 16(1), 152-162.

Niemeyer, G. ans Slotine, J.-J. (2004). Telemanipulation with Time Delays. International Journal of Robotics Research 23(9), 873-890.

Secchi, C., S. Stramigioli and C. Fantuzzi (2006). Position drift compensation in porthamiltonian based telemanipulation. In: Proceedings of IEEE/RSJ IROS. Beijing, China.

Stramigioli, S., A. van der Schaft, B. Maschke and C. Melchiorri (2002). Geometric scattering in robotic telemanipulation. IEEE Transactions on Robotics and Automation.

Stramigioli, S., C. Secchi, A.J. van der Schaft and C. Fantuzzi (2005). Sampled data systems passivity and discrete port-hamiltonian systems. IEEE Transactions on Robotics 21(4), 574-587.

van der Schaft, A.J. (2000). $L_{2}$-Gain and Passivity Techniques in Nonlinear Control. Communication and Control Engineering. Springer Verlag. 